\title{
Las representaciones sociales sobre el cambio climático de los estudiantes de pedagogía en México: un acercamiento desde la perspectiva de género
}

\author{
RAÚL CALIXTO FLORES \\ Universidad Pedagógica Nacional, Ajusco - México \\ Recibido el 22-08-2017; primera evaluación el 11 -11-2018; \\ segunda evaluación el 28-11-2018; aceptado el 12-12-2018
}

\section{RESUMEN}

En este artículo se describen los resultados de una investigación desarrollada con estudiantes de pedagogía de la Universidad Nacional Autónoma de México y de la Universidad Pedagógica Nacional de México con el objetivo de identificar las representaciones sociales que poseen sobre el cambio climático; se utilizó la entrevista, la encuesta con un cuestionario semiestructurado y un cuestionario con escala tipo Likert y una carta asociativa. En los resultados obtenidos se identifica la existencia de representaciones sociales del cambio climático; con características semejantes entre los estudiantes (femenino y masculino) y actitudes más favorables en las estudiantes (femenino). En general, los componentes de las dimensiones de las representaciones sociales son semejantes por género y por universidad. En las representaciones predominan la información sobre los efectos del cambio climático y el reconocimiento de la importancia de la educación ambiental.

Palabras clave: cambio climático, educación ambiental, pedagogía, representación social.

\footnotetext{
* Doctor en Pedagogía por la Universidad Nacional Autónoma de México, integrante de la Academia Nacional de Educación Ambiental, del Consejo Mexicano de Investigación Educativa, de la Red Durango de Investigadores Educativos y del Consejo Mexicano de Educación Internacional para la Integración y Desarrollo de las Naciones. Miembro del Sistema Nacional de Investigadores. Profesor/ investigador del Área académica 2. «Diversidad e Interculturalidad» en la Universidad Pedagógica Nacional, Ajusco. Docente en la línea en educación ambiental de la maestría en desarrollo educativo y de la licenciatura en pedagogía. Correo: calixto07@gmail.com
} 
The social representations on climate change of the students of pedagogy in Mexico: An approach from the perspective of gender

\section{Abstract}

This article describes the results of a research carried out with students of pedagogy of the National Autonomous University of Mexico and of the National Pedagogical University, of Mexico in order to identify the social representations they have about climate change; we used the interview, the survey with a semi-structured questionnaire and a questionnaire with Lickert-type scale and an associative letter. The obtained results the existence of social representations of the climate change; with similar characteristics between the students (female and male) and more favorable attitudes in the students (female). In general, the components of social representations dimensions are similar by gender and by university. In the representations, information on the effects of climate change and the recognition of the importance of environmental education predominate.

Keywords: climate change, environmental education, pedagogy, social representation

As representaçóes sociais sobre mudança climática dos estudantes de pedagogia no México: uma abordagem na perspectiva de gênero

Resumo

Este artigo apresenta os resultados da pesquisa realizada com estudantes da Universidade Nacional Autônoma do México e Universidade Nacional Pedagógica do México com o objetivo de identificar as representaçóes sociais têm sobre as alteraçóes climáticas; a entrevista foi usado, o questionário do inquérito e um questionário semi-estruturado com escala de Likert e um carta associativa. Nos resultados da identificado existência do as representaçóes sociais têm sobre as alteraçóes climáticas; os estudantes (feminino e masculino) e atitudes mais favoráveis nos estudantes (feminino). Em geral, os componentes das dimensóes das representaçóes sociais são semelhantes por gênero e universidade. Nas representaçôes predominam informaçóes sobre os efeitos das alteraçôes climáticas e reconhecimento da importância da educação ambiental.

Palavras-chave: mudança climática, a educação ambiental, pedagogia, representação social. 


\section{INTRODUCCIÓN}

El cambio climático (CC) es resultado de la combinación de diversos fenómenos, originados principalmente por el uso excesivo de combustibles fósiles que dan lugar a la emisión y acumulación de los gases con efecto invernadero que tiene efectos negativos en el ambiente.

El planeta se encuentra en una crisis del «no retorno» (Kates, 2007); es decir, los efectos del CC son irreversibles. Sin embargo, en la sociedad no se perciben estos efectos porque no se tiene presente una vivencia colectiva. En este sentido, la educación ambiental puede contribuir a hacer visible lo que en la sociedad no se observa y atenta contra el planeta, y por ende, contra la vida. El CC, como tema de interés público, es difundido cada vez más por los medios de comunicación y abordado en la mayoría de las instituciones de educación superior.

En este artículo se describen los principales resultados de una investigación en educación ambiental, realizada con estudiantes de la licenciatura en Pedagogía de la Universidad Nacional Autónoma de México (UNAM) y de la Universidad Pedagógica Nacional (UPN) de México, que tuvo como objetivo identificar y caracterizar las representaciones sociales (RS) del CC. En el análisis de resultados, se emplea la perspectiva de género para identificar las diferencias entre las representaciones de las mujeres y de los hombres.

\section{REFERENTES TEÓRICOS}

\subsection{Aproximaciones al concepto de género}

Espejel, Flores y Castillo (2014), señalan la relevancia del género como categoría analítica para diferenciar las relaciones que las mujeres y los varones establecen entre sí, con los recursos naturales y los ecosistemas; es decir, uno de los factores que influyen en la conformación de las representaciones con el ambiente son los roles y valores asignados para cada sexo en una sociedad determinada.

Los estudiantes de educación superior constituyen un sector de la población informado sobre el CC, y con una serie de representaciones que son necesarias conocer; representaciones que han ido conformándose principalmente por la influencia de los medios de comunicación y las relaciones sociales que se configuran de forma cotidiana. Una de las licenciaturas con mayor vinculación con el estudio del CC desde la perspectiva de la educación ambiental es la licenciatura de Pedagogía, es por ello que la presente investigación se aboca a estudiar en una muestra de estudiantes de pedagogía las RS que poseen sobre el CC. 
La licenciatura en Pedagogía es una carrera en la que predominan las estudiantes (femenino), ya que en la sociedad se le relaciona con el trato de los niños; es por ello que en esta investigación se ha incorporado la perspectiva de género, como una categoría analítica para describir las representaciones de las mujeres y de los varones que estudian esta licenciatura.

Esta perspectiva es escasamente utilizada en la investigación de las RS, entendida como una:

...posición teórica y metodológica que remite a comprender a la categoría de género como una construcción sociocultural que define lo socialmente aceptado y apropiado para cada sexo en una sociedad determinada, e incluye una serie de valores, roles y comportamientos diferenciados para hombres y para mujeres (Espejel, Flores y Castillo, 2014, p. 14).

El género no es sinónimo de mujer, sino una categoría analítica, que intenta subrayar el carácter construido de la dif erencia sexual en las relaciones sociales. El género, de acuerdo a Martínez (2003) «...es una dimensión básica de la diferenciación social que afecta las experiencias, intereses y capacidades de las personas; esto también referido a la gestión y el manejo de recursos naturales» (p. 226); es decir, el género cuestiona las relaciones femeninas y masculinas establecidas en una sociedad patriarcal en torno al ambiente, conlleva así una reflexión profunda en la organización social, económica y política, que trasciende al ámbito educativo.

Las relaciones femeninas-masculinas son constitutivas y constituyentes de las relaciones sociales, en donde se observa el predominio masculino, que se hace presente a través de los vínculos, estas relaciones condicionan la situación de la mujer «sistema sexo-género».

El género, como marco de análisis de la realidad, posibilita reconocer la subjetividad social de los papeles asignados a las mujeres y varones, la complejidad que se manifiesta en la realidad y la falsa homogeneización de lo heterogéneo. Con ello, se abre la posibilidad, de negar la legitimación de las diferencias inequitativas entre los géneros y realizar una construcción teórica de la perspectiva femenina de la realidad social.

La categoría de género resulta importante para plantear al ambiente desde una perspectiva diferente a la masculina, en la cual se reconozca el protagonismo de la mujer en los distintos fenómenos sociales. Género pasa a ser una forma de denotar las construcciones culturales, la creación totalmente social de las ideas sobre los roles apropiados para mujeres y varones. Es una forma de referirse a los orígenes exclusivamente sociales de las identidades subjetivas de mujeres y varones. 


\subsection{Las representaciones sociales}

Las RS centran su atención en el conocimiento de sentido común, el conocimiento que se comunica de forma cotidiana; tiene su origen en el conocimiento científico, el cual es utilizado para explicar los fenómenos de distinta naturaleza que ocurren en la cotidianidad.

Cada sujeto construye sus representaciones en un proceso de actividad cultural, ligado a diversos procesos cognitivos. La construcción de las RS está fuertemente relacionada con las prácticas culturales que el sujeto realiza en su grupo.

En tanto que fenómenos, las representaciones sociales se presentarán bajo formas más o menos complejas. Son imágenes que condensan un conjunto de significados: sistemas de referencia que nos permiten interpretar lo que nos sucede, e incluso, dar un sentido a lo inesperado; categorías que sirven para clasificar las circunstancias, los fenómenos y a los individuos con quienes tenemos algo que ver, teorías que permiten establecer hechos sobre ellos. Y a menudo, cuando se les comprende dentro de la realidad concreta de la vida social, las representaciones sociales son todo ello junto (Jodelet, 1984, p. 472).

Las RS se encuentran en la parte subjetiva de los sujetos, están presentes en su pensamiento y se manifiestan en las acciones cotidianas, de fenómenos globales como el CC.

\subsection{Las representaciones sociales del cambio climático}

En cuanto al CC, se han acrecentado sus efectos sobre la Tierra, debido al creciente aumento de la emisión de los gases de efecto invernadero. Delgado (2015) refiere que Paul Jozef Crutzen introdujo en el año 2000 el término antropoceno. Las afectaciones al planeta originadas por las actividades humanas son de tal magnitud que los dańos en el ambiente son irreversibles.

El CC antropogénico alude a lo que se deriva de las actividades humanas que aceleran el proceso de calentamiento global, generando efectos negativos en las dimensiones ecológicas, sociales y económicas del ambiente. Muchos de los cambios provocados son irreversibles en distintas regiones del planeta.

En este marco, se plantea en este escrito que la investigación de las RS del CC en las instituciones de educación superior proporcionan elementos para comprender si la educación ambiental ha promovido en los estudiantes el reconocimiento del origen y efectos del CC; así como las múltiples alternativas de prevención, mitigación o adaptación que pueden generar por medio de su desarrollo académico y profesional. La educación ambiental es entendida como una dimensión de la educación contemporánea que se preocupa por 
optimizar la red de relaciones persona-grupo social-ambiente (Sauvé, 1997). En este sentido, la investigación sobre RS de diversos tópicos, como el cambio climático, aporta información relevante para la educación ambiental, además de conocimientos sobre los sentidos y significados de estas relaciones.

Las RS del CC en estudiantes de educación superior han sido identificadas en distintas poblaciones de estudiantes, por diferentes autores, entre otros Cabecinhas, Lázaro y Carvalho (2008); González Gaudiano y Maldonado (2012); Meira y Arto (2013); Terrón y Bahena (2015). Los resultados demuestran la presencia de RS sobre CC en los estudiantes universitarios; es decir, el CC ha pasado a formar parte del conocimiento de sentido común en este sector de la población. Con estas representaciones, los estudiantes dialogan, explican y toman decisiones en su vida cotidiana.

\section{OBJETIVOS Y MÉTODo}

El objetivo central del estudio fue el de identificar y caracterizar los contenidos de las RS del CC que tienen los estudiantes de pedagogía, a partir del cual se plantearon dos objetivos secundarios.

- Explorar los componentes de las RS sobre las causas, efectos y alternativas de solución del CC y

- Analizar las posibles diferencias en los componentes de las representaciones por el género de los estudiantes.

La investigación realizada se inscribe en el enfoque procesual de las RS, se privilegia el manejo de información cualitativa, ya que interesó descubrir los significados no manifiestos de la experiencia de los estudiantes. Es un estudio exploratorio y descriptivo, congruente con el enfoque procesual de las RS (Banchs, 2000). El diseño metodológico se orientó hacia la identificación y caracterización de las representaciones presentes en los estudiantes por medio de diversos instrumentos: cuestionarios con preguntas abiertas y cerradas (Meira, 2002) y cartas asociativas, fundamentas en la asociación libre de palabras a partir de un término inductor, los sujetos expresan las palabras libremente (Abric, 1994). Los instrumentos empleados se pilotearon previamente con una muestra reducida de estudiantes de la licenciatura en Pedagogía de la UNAM. Esta investigación desarrolla un proceder metodológico ya trabajado en estudios previos (Calixto, 2008).

La muestra fue selectiva, con base en una invitación personal a los estudiantes de dos grupos de pedagogía del séptimo semestre, uno de cada universidad. En la investigación participaron 20 estudiantes de la UNAM, 12 
(femenino) y 8 (masculino); también colaboraron 23 estudiantes de la UPN, 18 (femenino) y 5 (masculino).

La licenciatura en Pedagogía es una carrera en la que predominan las estudiantes (femenino), en el caso del grupo de estudio de la UNAM, el porcentaje de las estudiantes (femenino) del 60\% y en el grupo de estudio de la UPN, el porcentaje de las estudiantes (femenino) es de $78,2 \%$.

En cuanto al promedio de edad, también se observa una diferencia entre las dos instituciones: en la UNAM el promedio de edad de las estudiantes (femenino) es de 24,1 años y el promedio en los estudiantes (masculino) es de 27,1 ańos; en la UPN, el promedio de edad de las estudiantes (femenino) es de 23,7 años, en tanto que en los estudiantes (masculino) es de 28,8 años. Esta diferencia obedece a varios factores, pero el principal, referido por los propios estudiantes (varones), es el de que la carrera de pedagogía no fue su primera opción de estudios.

\section{Resultados Y Discusión}

En los resultados se identifica la existencia de las RS, y las características de los contenidos que las conforman.

Moscovici (1979) remite a la dimensión de información la suma de conocimientos poseídos de un objeto social, a su cantidad y a su calidad, más o menos estereotipada, trivial u original. La dimensión de información se constituye con los conocimientos que poseen los sujetos sobre un objeto. La información se construye y se reconstruye, es la relación que define el proceso cognoscitivo. En los sujetos existe una gran cantidad de información que comprende una gran variedad de conocimientos.

Los estudiantes de la UPN refieren que, en su formación universitaria, no han cursado una asignatura relacionada con la educación ambiental o el CC, caso contrario de los estudiantes de la UNAM, quienes hacen mención de la asignatura de "Taller de Educación No Formal» (Educación y Medio Ambiente). Los jóvenes de ambas instituciones hacen mención que, en algunas clases, los profesores llegan a comentar algunos temas referidos a la educación ambiental y que se relacionan con el CC.

\subsection{Información y campo representacional}

La mayoría de los estudiantes utiliza como principal fuente de información el internet; este medio de comunicación cada vez adquiere mayor importancia entre los jóvenes; el porcentaje es muy semejante en los estudiantes de ambas 
instituciones. Estos datos difieren de los obtenidos por Meira (2013), ya que encuentra, en la sociedad española, como principales fuentes de información en personas entre 18 y 24 años, la televisión, las campañas publicitarias y las «clases».

La televisión en los jóvenes universitarios de la UNAM y UPN ocupa en la mayoría de los casos un lugar secundario. De acuerdo a los datos obtenidos, un porcentaje mayor de los estudiantes (masculino) usan más que las estudiantes (femenino) la televisión como fuente de información sobre el CC, y se observan porcentajes más altos en los estudiantes (masculino) de la UPN.

Las respuestas respecto a la influencia que han recibido de algunas personas para conocer más sobre el CC son muy semejantes en ambos géneros y en ambas instituciones. Los amigos y los profesores son las personas que más influyen en los estudiantes. Estas fuentes pueden ser poco confiables, si no cuentan con la información adecuada del CC; para los estudiantes de la UPN, también de un científico se obtienen porcentajes elevados. En tanto que González-Gaudiano y Maldonado (2014) identifican que los estudiantes de la Universidad Veracruzana obtienen la información principalmente de los científicos, los grupos ecologistas y los educadores ambientales. Y los políticos y empresarios son las personas que influyen en menor medida en los estudiantes. Las respuestas son muy similares entre los estudiantes de ambos géneros. Los estudiantes refieren diferentes aspectos asociados con el CC.

No se observan diferencias entre los estudiantes de ambas instituciones; los estudiantes tienen un similar conocimiento de diversos aspectos relacionados con el CC; aspectos como los sumideros de carbono y el comercio de emisiones de carbono son desconocidos para la mayoría de los estudiantes. La protección de los sumideros de carbono y el comercio de emisiones de carbono son estrategias instrumentadas para propiciar la sustentabilidad del planeta, y con ello mitigar los efectos del CC. Esta información aún no está presente en las RS de los estudiantes.

A diferencia de la información de las RS del CC de los estudiantes de otras instituciones, referidas en otras investigaciones (Boyes y Stanisstreet, 1992; Dove, 1996; Rebich y Gautier, 2005; Correa, 2012), los estudiantes de la UPN incorporan a la educación ambiental y a la sustentabilidad, aspectos que se relacionan más con la licenciatura en Pedagogía que cursan en la Universidad.

Respecto a la importancia que se le ha dado al CC en los distintos medios de comunicación, los estudiantes en su gran mayoría coinciden en que se le ha dado una menor importancia, el 100\% de los estudiantes (masculino) de ambas instituciones, así como el 91,66\% de las estudiantes (femenino) de la UNAM y $83,3 \%$ de las estudiantes (femenino) de la UPN son los porcentajes más altos. 
En el estudio realizado, se identifica que un porcentaje reducido de las estudiantes (femenino) de ambas instituciones coinciden en indicar que al CC se le ha dado la importancia que tiene, señalamiento que no es compartido por los estudiantes (masculino). Existen dudas en el caso de las estudiantes (femenino) sobre la importancia real del CC. Los datos obtenidos coinciden con los obtenidos por Meira (2013) en una muestra de personas jóvenes, en los que predomina también la percepción de una subvaloración de la importancia del CC.

En cuanto a la identificación del origen del CC, no se observan diferencias en las respuestas por el género de los estudiantes, pero si, por la institución; en las estudiantes (femenino) el porcentaje más alto $(58,3 \%)$ corresponde al de las estudiantes (femenino) de la UNAM, y en los estudiantes (masculino) el porcentaje más alto $(60 \%)$ se encuentra en la UPN.

Si bien el CC es un proceso natural de la Tierra, en los últimos años, se ha acrecentado su duración, como consecuencia de la extracción y uso intensivo de los combustibles fósiles (origen antropogénico). Son pocos los estudiantes (femenino y masculino) de la UPN que identifican la influencia combinada de ambos procesos. También se observa en los estudiantes (femenino y masculino) un desconocimiento de las causas naturales del CC. Resultados similares han sido reportados por Meira y Arto (2014). En el caso de la UPN, la mayor proporción de estudiantes identifican el origen del CC por causas humanas.

Las respuestas de los estudiantes (femenino y masculino) de ambas instituciones son semejantes; la información más conocida ha sido abordada con mayor tiempo por los distintos medios de comunicación, y otra información, que es casi desconocida, solo se difunde en algunos espacios de las universidades. Así, por ejemplo, los estudiantes cuentan con mayor información sobre el Protocolo de Kioto (75\% — femenino- y 44,4\% — masculino- de la UNAM) y sobre la Estrategia Nacional de Educación Ambiental (77,7\% —femenino- y $80 \%$ de los estudiantes — masculino- de la UPN). Los estudiantes de ambas instituciones cuentan con menor información sobre los objetivos del milenio de la Organización de las Naciones Unidas (16,6\% femenino-y 11,1\% — masculino- UNAM; 11,1\% - femenino- y 20\% - masculino- UPN). En algunos rubros, existe una diferencia significativa entre los estudiantes por el género e institución, por ejemplo, del Programa Universitario del Medio Ambiente (programa ambiental de la UNAM) es conocido por un poco más de la mitad de las estudiantes (femenino), pero no así los estudiantes (masculino). En tanto que, sobre la Convención Marco de las Naciones Unidas sobre el Cambio Climático, el 61,1\% de las estudiantes (femenino) de la UPN tienen alguna información, solo el $20 \%$ de los 
estudiantes (masculino) puede identificar esta convención, y esta situación ocurre en forma opuesta cuando se aborda el Programa de las Naciones Unidas por el Medio Ambiente, solo el 11,1\% de las estudiantes (femenino) lo identifica, en tanto que el $80 \%$ de los estudiantes (masculino) lo conoce.

La información que poseen los estudiantes de la UNAM y UPN sobre los organismos y/o instituciones que promueven acciones ante el CC es muy semejante. Greenpeace es la organización más conocida por los estudiantes de las dos universidades (100\% de las estudiantes (femenino) y $88.8 \%$ de los estudiantes (masculino) de la UNAM; 94,4\% de las estudiantes (femenino) y $60 \%$ de los estudiantes (masculino) UPN, por la continuidad y difusión de sus acciones a favor del medio ambiente.

Por otra parte, se observa que los estudiantes utilizan términos similares al referirse al CC. En la tabla 1, se aprecia que el término «muerte» es uno de los más referidos por los estudiantes (femenino-masculino) de la UNAM; en tanto que en los estudiantes (femenino-masculino) de la UPN el término más utilizado es el de "contaminación». No se observa, en las respuestas, alguna diferencia significativa por el género de los estudiantes.

Tabla 1. Términos más utilizados al pensar en CC

\begin{tabular}{lcccc}
\hline Términos & $\begin{array}{c}\text { UNAM- } \\
\text { Fem } \%\end{array}$ & $\begin{array}{c}\text { UNAM- } \\
\text { Masc\% }\end{array}$ & $\begin{array}{c}\text { UPN- } \\
\text { Fem } \%\end{array}$ & $\begin{array}{c}\text { UPN- } \\
\text { Masc\% }\end{array}$ \\
\hline Calor & 41,6 & 37,5 & 44,4 & 80,0 \\
Contaminación & 41,6 & 50,0 & 61,1 & 80,0 \\
Calentamiento & 41,6 & 75,0 & 16,6 & 40,0 \\
Muerte & 41,6 & 87,5 & 27,7 & 60,0 \\
Sequía & 41,6 & 75,0 & 27,7 & 80,0 \\
Extinción & 16,6 & 50,0 & 11,1 & 60,0 \\
Basura & 16,6 & 25,0 & 38,8 & 40,0 \\
\hline
\end{tabular}

Fuente: Encuesta de investigación. Elaboración propia.

Los términos incluidos en la tabla 1 dan cuenta del núcleo figurativo de las RS del CC en los estudiantes de la licenciatura en Pedagogía de ambas instituciones. A partir de estos términos, los estudiantes utilizan otras palabras con las cuales comunican lo que les significa el CC; otras palabras se asocian y constituyen el núcleo figurativo de las RS. Al momento de identificar las relaciones entre los componentes del núcleo figurativo se objetiva el campo representacional. 
En la figura 1, se observan las relaciones más frecuentes señaladas por las estudiantes y la configuración del núcleo figurativo, en el que es posible observar el predominio de la identificación de los efectos sobre las causas del CC.

En la figura 2, se observa la integración del núcleo figurativo con elementos relacionados con los efectos del CC, que conducen a la destrucción o la extinción.

\section{Figura 1. Campo representacional. UNAM-Pedagogía-Femenino}

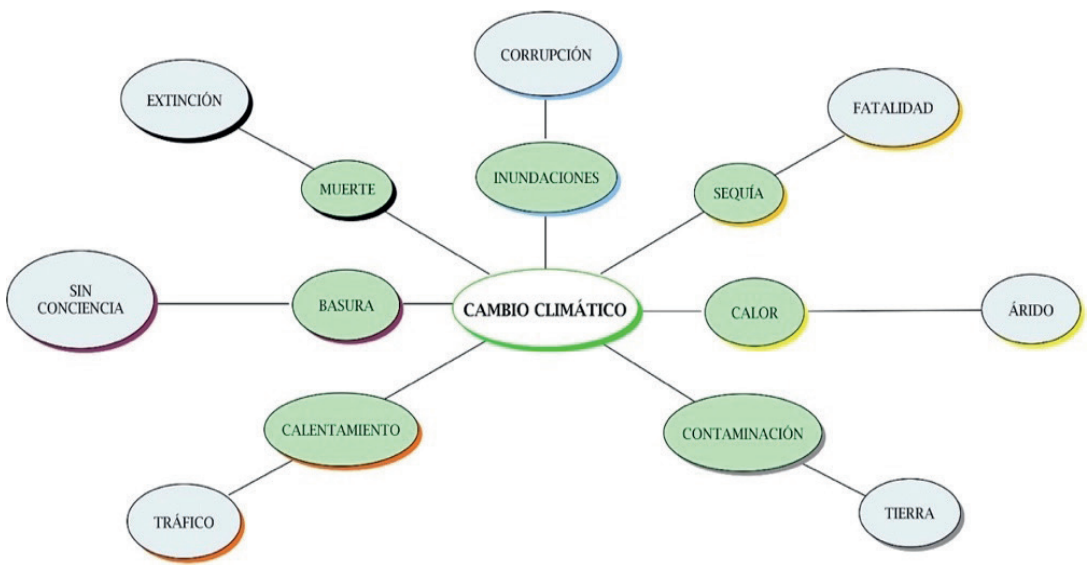

Fuente: Elaboración propia con base en los resultados de la carta asociativa.

\section{Figura 2. Dimensión del campo representacional. UNAM-Pedagogía-Masculino}

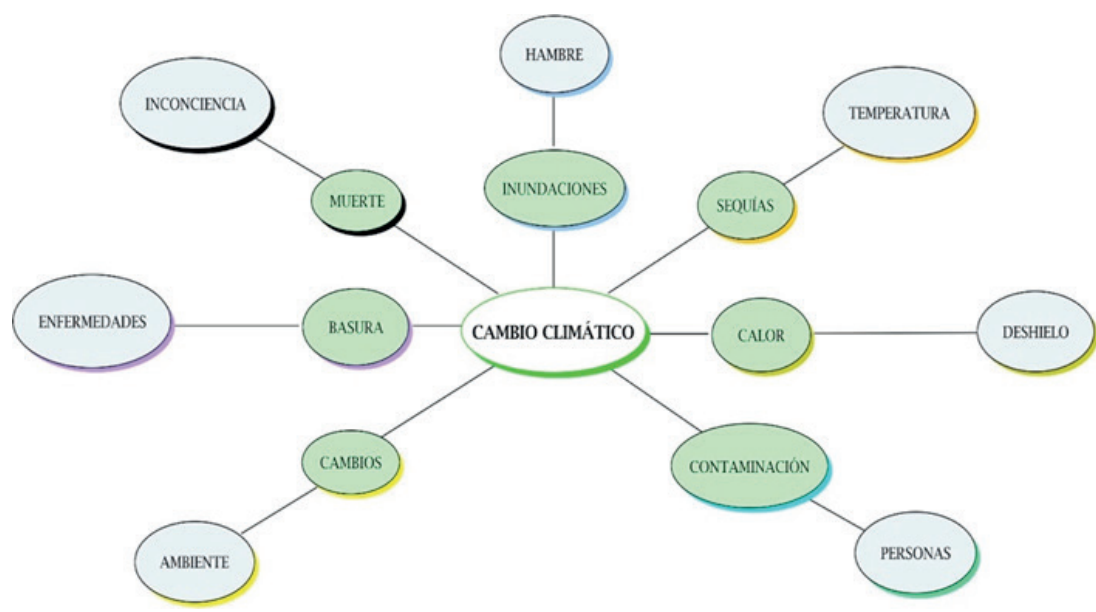

Fuente: Elaboración propia con base en los resultados de la carta asociativa. 
En la figura 3, se puede identificar que las estudiantes reconocen los efectos del CC al medio ambiente y a los seres humanos; incorporan un mayor número de aspectos sociales.

Los términos del campo de representación que se muestran en la figura 4 forman asociaciones en los que predominan los efectos del CC sobre los seres humanos.

Figura 3. Dimensión del campo representacional. UPN-Pedagogía-Femenino

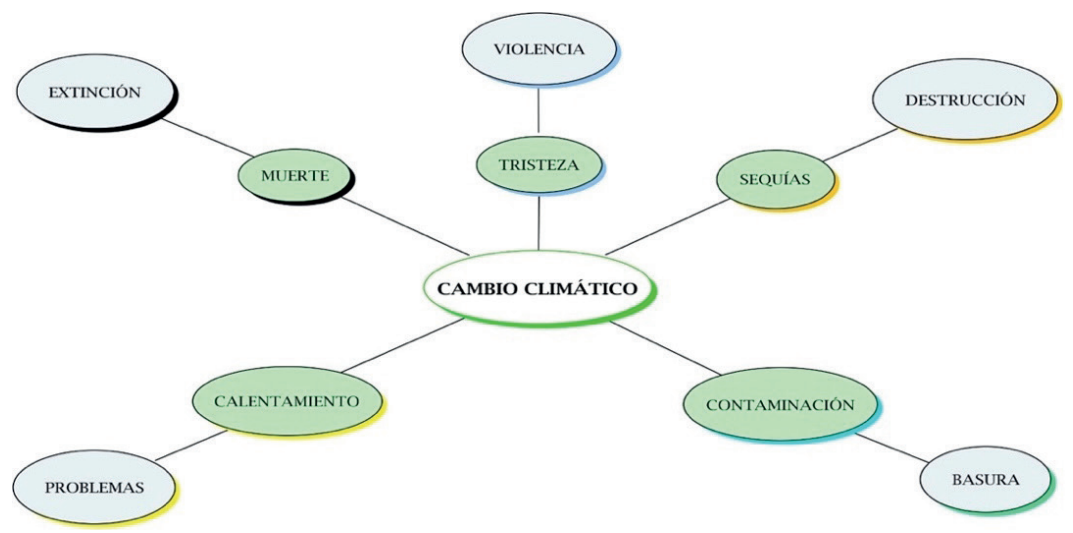

Fuente: Elaboración propia con base a os resultados de la carta asociativa.

Figura 4. Dimensión del campo representacional. UPN-Pedagogía-Masculino

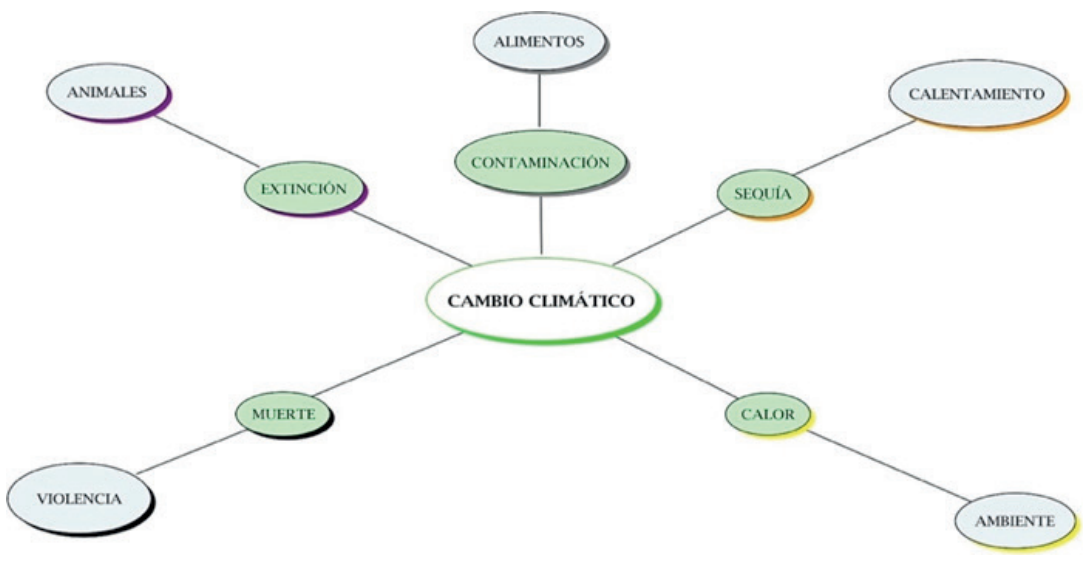

Fuente: Elaboración propia con base a los resultados de la carta asociativa. 
Las estudiantes de ambas instituciones toman en cuenta algunos aspectos sobre las causas del CC: inconciencia o sin conciencia y corrupción. En el caso del campo de representación en los estudiantes (masculino), tienen presente a la violencia y a la muerte.

En los campos de representación de los estudiantes, no se encuentran elementos referidos a las alternativas de solución, o referidas a las acciones de prevención, mitigación o adaptación. Al igual que en las RS de los estudiantes de otras licenciaturas, en el estudio realizado por González-Gaudiano y Maldonado (2014), se observa el predomino del uso de palabras vinculadas a los efectos del CC.

\subsection{Las actitudes}

En la teoría de las RS, las actitudes son consideradas como una dimensión que articula a las otras dimensiones para darle un sentido a la representación (Calixto, 2008). En las siguientes tablas se muestran los porcentajes de algunas de las principales actitudes manifestadas por los estudiantes sobre el CC. En las tablas de actitudes, se usan las siguientes siglas: $\mathrm{MD}=$ muy en desacuerdo, $\mathrm{D}=$ desacuerdo, $\mathrm{DA}=$ ni desacuerdo, ni acuerdo, $\mathrm{A}=$ acuerdo, $\mathrm{MA}=$ muy acuerdo.

Existen pocas diferencias en las respuestas de los estudiantes de ambas instituciones; son semejantes los porcentajes de estudiantes (femenino y masculino). Por una parte, la consideran como una gran amenaza y, por otra, no están de acuerdo en esta opinión. Los porcentajes más altos a favor son de las estudiantes (femenino) de la UPN y los porcentajes más bajos son los de las estudiantes (femenino) de la UNAM.

Tabla 2. CC como la mayor amenaza que enfrenta la vida

\begin{tabular}{lccccc}
\hline Institución & MD\% & D\% & DA\% & A\% & MA\% \\
\hline UNAM-Femenino & 8,3 & 33,3 & 33,3 & 16,6 & 8,3 \\
UNAM-Masculino & 0,0 & 33,3 & 22,2 & 44,4 & 0,0 \\
UPN-Femenino & 11,1 & 5,5 & 5,5 & 55,5 & 22,2 \\
UPN-Masculino & 20,0 & 20,0 & 0,0 & 40,0 & 20,0 \\
\hline
\end{tabular}

Fuente: Elaboración propia con base en los resultados de la escala Likert. 
Tabla 3. El CC es un problema del futuro, no del presente

\begin{tabular}{lccccc}
\hline Institución & MD\% & D\% & DA\% & A\% & MA\% \\
\hline UNAM-Femenino & 50,0 & 41,6 & 8,3 & 0,0 & 0,0 \\
UNAM-Masculino & 22,2 & 33,3 & 0,0 & 33,3 & 0,0 \\
UPN-Femenino & 38,3 & 27,7 & 0,0 & 22,2 & 11,1 \\
UPN-Masculino & 40,0 & 20,0 & 0,0 & 20,0 & 20,0 \\
\hline
\end{tabular}

Fuente: Elaboración propia con base en los resultados de la escala Likert.

Al cuestionar a los estudiantes sobre el CC como un problema del futuro, la mayoría de los estudiantes manifiesta estar en desacuerdo.

En las estudiantes (femenino) de la UNAM, se identifica con mayor claridad la posición de desacuerdo; en tanto que, en los estudiantes (masculino) de la UNAM y de la UPN, las respuestas están divididas, no predomina el acuerdo o desacuerdo. Datos similares son referidos por Meira (2013), en un tercio aproximadamente de la población estudiada $(35,8 \%)$ lo remiten al futuro.

Tabla 4. No le influyen mis acciones, porque es un problema demasiado grande

\begin{tabular}{lccccc}
\hline Institución & MD\% & D\% & DA\% & A\% & MA\% \\
\hline UNAM-Femenino & 66,6 & 41,6 & 0,0 & 0,0 & 0,0 \\
UNAM-Masculino & 22,2 & 44,4 & 22,2 & 22,2 & 0 \\
UPN-Femenino & 61,1 & 16,6 & 11,1 & 5,5 & 5,5 \\
UPN-Masculino & 60,0 & 20,0 & 0,0 & 20,0 & 0,0 \\
\hline
\end{tabular}

Fuente: Elaboración propia con base en los resultados de la escala Likert.

La posición en desacuerdo es más definida en las estudiantes (femenino) de ambas instituciones, mientras que en los estudiantes (masculino) no se observa una posición bien definida, se encuentran $22,2 \%$ en acuerdo en la UNAM y del $20 \%$ en la UPN. Estos resultados son similares a los que se reportan en algunos sectores de la población de países como Turquía, Rumania, Bulgaria y Lituania (Dirección General de Comunicación de la Comisión Europea, 2013).

En cuanto a la postura que asumen los estudiantes respecto a si el CC no les afecta personalmente, en su mayoría están en desacuerdo. 
Tabla 5. El CC no me afecta personalmente

\begin{tabular}{lccccc}
\hline Institución & MD\% & D\% & DA\% & A\% & MA\% \\
\hline UNAM-Femenino & 50,0 & 41,6 & 8,3 & 0,0 & 0,0 \\
UNAM-Masculino & 11,1 & 55,5 & 0,0 & 11,1 & 11,1 \\
UPN-Femenino & 83,3 & 11,1 & 0,0 & 5,5 & 0,0 \\
UPN-Masculino & 80,0 & 0,0 & 0,0 & 20,0 & 20,0 \\
\hline
\end{tabular}

Fuente: Elaboración propia con base en los resultados de la escala Likert.

Un mayor número de estudiantes (femenino) de la UNAM y de la UPN y de estudiantes (masculino) de la UPN reconocen que el CC les afecta personalmente. Los porcentajes más altos se encuentran en las estudiantes (femenino) de la UPN. Al vincularse con aspectos personales, la postura es más definida en las estudiantes mujeres de ambas instituciones y en los estudiantes (masculino) de la UPN. Se observa, en los estudiantes, la existencia de un alto nivel de preocupación y por los riesgos derivados del CC, al igual que en las investigaciones de Cabecinhas, Lázaro y Carvalho (2008).

Respecto a la posición ante la relación de la educación ambiental con el CC, la mayoría de los estudiantes manifiesta estar de acuerdo en la importancia que tiene para el cambio de comportamientos.

Tabla 6. La educación cambia los comportamientos que influyen en el CC

\begin{tabular}{lccccc}
\hline Institución & MD\% & D\% & DA\% & A\% & MA\% \\
\hline UNAM-Femenino & 0,0 & 0,0 & 16,6 & 41,6 & 41,6 \\
UNAM-Masculino & 0,0 & 22,2 & 11,1 & 33,3 & 33,3 \\
UPN-Femenino & 11,1 & 0,0 & 22,2 & 22,2 & 44,4 \\
UPN-Masculino & 0,0 & 0,0 & 20,0 & 20,0 & 60,0 \\
\hline
\end{tabular}

Fuente: Elaboración propia con base en los resultados de la escala Likert.

Los porcentajes más altos de todos los subgrupos de estudiantes se encuentran en la posición de estar muy de acuerdo; también se identifican estudiantes (masculino) de la UNAM y estudiantes (femenino) de la UPN, que están en desacuerdo. Al igual que en otras investigaciones, persisten barreras y disposiciones no favorables en los sujetos para actuar ante el CC (González-Gaudiano 
y Maldonado, 2012; Montoya y Acevedo, 2013; Jaspal, Nerlich y Cinnirella, 2014; Ramírez, 2014).

En términos generales, se observa la existencia de una actitud favorable de los estudiantes en reconocer la importancia del CC, por los efectos que tiene para el planeta, y por ende para la biodiversidad y las sociedades humanas, resultados que pueden ser tomados en cuenta al elaborar una propuesta en educación ambiental.

La educación ambiental ante el CC ha de trascender del plano informativo al afectivo (Gould, 1991) para lograr cambios significativos en el comportamiento favorable al ambiente.

En los diversos componentes que conforman las RS del CC, se observan diferencias entre las actitudes entre las estudiantes (femenino) y los estudiantes (masculino). Existe una actitud más favorable en las estudiantes (femenino) por actuar frente al CC.

\section{Conclusiones}

En los resultados obtenidos, se identifica la existencia de RS del CC, con características semejantes entre las estudiantes (femenino) y los estudiantes (masculino) con actitudes más favorables en las mujeres. También se identifican, en las estudiantes (femenino) de ambas instituciones, en el campo representacional, un vínculo mayor entre el CC y los aspectos sociales.

Por otra parte, se hace evidente, en la dimensión del campo representacional y la dimensión de actitudes, un componente afectivo en las RS que puede influir sobre la actitud de los estudiantes en relación al (CC). Los estudiantes de pedagogía, a diferencia de los estudiantes de otras disciplinas, evidencian la importancia de la educación ambiental y de la sustentabilidad ante los problemas derivados del CC.

La dinámica de cada una de las instituciones y el acceso de fuentes digitales de información han posibilitado en los estudiantes la construcción de las RS del CC que se caracterizan por el predominio de información de los efectos y la existencia de escasos elementos sobre las causas económicas y/o políticas del CC.

Por lo general, en las investigaciones de RS no se emplea la perspectiva de género. Esta resulta relevante para evidenciar el tratamiento inequitativo de las políticas sociales para resolver los problemas ambientales, aunque es necesario seńalar que en el ámbito educativo no se puede generalizar, ya que en los últimos años en las estudiantes ha aumentado la conciencia del origen de estas desigualdades. El «Grupo Intergerencial de Género» plantea, en una 
de sus fichas informativas, la importancia de incorporar a las mujeres en la generación de políticas de adaptación y mitigación al CC.

Estas políticas, por lo general, reproducen una visión patriarcal del tratamiento de los problemas ambientales, que reproduce como "naturales», diversas actividades, emociones y comportamientos para las mujeres y otras para los varones y un reconocimiento social diferenciado. De esta forma, se reproduce una diferencia asimétrica de las relaciones entre los géneros; sin embargo, en el caso de las y los estudiantes universitarios de la UNAM y la UPN, se identifica un pensamiento social similar ante el CC, en ambos géneros existe un reconocimiento sobre las implicaciones desfavorables hacia el ambiente y las sociedades humanas.

Por otra parte, la ausencia de información social, política y económica en los estudiantes se refleja en la conformación de sus RS, en donde son escasas las referencias sobre las causas del CC, predominan en la dimensión de información sobre los efectos a las comunidades humanas.

Las RS del CC en los estudiantes universitarios están en configuración, por lo que son susceptibles de enriquecerse o de transformarse. Por ello, la educación ambiental puede construir estrategias que incidan en el discurso de la racionalidad instrumental y se planteen nuevas formas de mirar las relaciones de las mujeres y varones, entre sí y con el ambiente.

Por último, al realizar una valoración global de las RS identificadas, y tomando en cuenta las categorías de jóvenes respecto al ambiente (anti ambientalistas, indiferentes ambientales, pesimistas prácticos, activos individuales y activos organizados), propuestas por la Universidad Complutense de Madrid (2008), se puede decir que los estudiantes de la licenciatura en Pedagogía de la UNAM y de la UPN son activos individuales ya que manifiestan que realizan actividades cotidianas (pequeñas acciones) a favor del ambiente, pero sin llegar a constituir relaciones, «lazos» $\mathrm{o}$ «redes» con otros grupos de jóvenes.

Sin embargo, por medio de la educación ambiental se puede superar la idea de que son suficientes las pequeńas acciones para dar solución a los problemas ambientales; estas son muy importantes, pero se crea la falsa percepción de que de esta forma se solucionan los problemas ambientales sin tocar el origen de los mismos (metáfora del "granito de arena», González-Gaudiano y Maldonado, 2013); también la educación ambiental pueden contribuir a la desmitificación de que estas acciones le corresponden por su propia naturaleza solo a las mujeres.

Ante la crisis actual del medio ambiente, y del reconocimiento de las graves consecuencias de las actividades industriales y las actitudes antropocéntricas, se hace necesaria una educación ambiental desde la educación básica. La educación ambiental constituye una alternativa que incide en el cambio 
de prácticas. Para tal fin, el estudio de las RS proporciona elementos para comprender la importancia de los componentes afectivos en la toma de decisiones cotidianas. La identificación del anclaje de las RS del CC proporciona elementos para incidir en el cambio de actitud.

Los futuros pedagogos pueden contribuir a la educación en cuestiones ambientales, generando en los nińos, jóvenes y adultos una conciencia ambiental, que se traduzca en un comportamiento ambientalmente solidario, responsable y sustentable.

La formación de pedagogos en la educación ambiental adquiere mayor relevancia, ya que pueden fomentar en la sociedad nuevos valores, acciones y representaciones ante el CC.

\section{REFERENCIAS BIBLIOGRÁFICAS}

Abric, C. (coord.) (1994). Prácticas sociales y representaciones. México: Ediciones Coyoacán.

Banchs, M. A. (2000). Aproximaciones procesuales y estructurales al estudio de las representaciones sociales. Papers on Social Representations, 9, 1-15.

Boyes, E. y Stanisstreet, M. (1992) 'Students' perceptions of global warming'. International Journal of Environmental Studies, 42(4), 287-300. https:// doi.org/10.1080/00207239208710804

Cabecinhas, R., Lázaro, A. y Carvalho, A. (2008). Communicating Climate Change: Discourses, Mediations and Perceptions. Braga: Centro de Estudos de Comunicação e Sociedade, Universidade do Minho. Disponible en http://www.lasics.uminho.pt/ojs/index.php/climate_change10

Calixto, R. (2008). Representaciones sociales del medio ambiente. Perfiles Educativos, XXX(120), 33-62.

Correa, I. (2012). Cambio climático y representaciones sociales entre estudiantes de educación superior. En B. Ortiz y C. Velasco (coords.), La percepción social del cambio climático (pp. 108-122). México: Universidad Iberoamericana Puebla y Secretaría de Medio Ambiente y Recursos Naturales.

Delgado, G.C. (2015). Water and urban metabolism. Journal of Polical Ecology, 22, 98-114.

Dove, J. (1996). Student teacher understanding of the greenhouse effect, ozone layer depletion and acid rain. Environmental Education Research, 2(1), 89-100. https://doi.org/10.1080/1350462960020108

Espejel, A., Flores A. y Castillo, I. (2014). Estereotipos de género en la educación ambiental. En A. Espejel y A. Flores (coords.), Educación ambiental, género y competencias (pp. 11-36). México: Universidad Autónoma de Tlaxcala. 
González-Gaudiano E. y Maldonado L. (2012). Representaciones sociales y cambio climático, el caso de Veracruz. En B. Ortiz y C. Velasco (coords.), La percepción social del cambio climático (pp. 82-106), México: Universidad Iberoamericana Puebla y Secretaría de Medio Ambiente y Recursos Naturales.

González-Gaudiano E. y Maldonado, A. L. (2014). Los jóvenes universitarios y el cambio climático. México: Universidad Veracruzana.

González-Gaudiano E. y Maldonado, L. (2014). ¿Qué piensan, dicen y hacen los jóvenes universitarios sobre el cambio climático? Un estudio de representaciones sociales. Educar em Revista, 3, 35-55. https://doi. org/10.1590/0104-4060.38106

Jaspal, R., Nerlich, B. y Cinnirella, M. (2014). Human Responses to Climate Change: Social Representation, Identity and Socio-psychological Action. Environmental Communication, 8(1), 110-130.

Jodelet, D. (1984). La representación social: fenómenos, conceptos y teoría. En Serge Moscovici, tr. David Rosenbaum, Psicología Social II (pp. 469-493). Barcelona: Paidós.

Kates, Robert W. (2007) Foreword. En S. Moser y L. Dilling (eds.), Creating a Climate for Change. Communicating Climate Change and Facilitating Social Change (pp. xiii-xv). Cambridge: Cambridge University Press. https://doi. org/10.1017/CBO9780511535871.002

Martínez, B, (2003). Empoderamiento y cambios en el manejo y control de recursos. En E. Tunón (coord.), Género y medio ambiente (pp. 221-254), México: Ecosur, Semarnat-Plaza y Valdes.

Meira, P.Á. (2002). Problemas ambientales globales y educación ambiental: una aproximación desde las representaciones sociales del cambio climático. En M. Campillo (ed.), El papel de la educación ambiental en la pedagogía social (pp. 31-133). Murcia: Diego Marín.

Meira, P.Á. (dir.) (2013). La respuesta de la sociedad española ante el cambio climático. Madrid: Fundación Mapfre y Aldine editorial.

Meira, P.Á. y Arto, M. (2014). Representaciones del cambio climático en estudiantes universitarios en España: aportes para la educación y la comunicación. Educar em Revista, 3, 15-33. https://doi.org/10.1590/0104-4060.38041

Montoya, E. y Acevedo, E. (2013). Preocupación ambiental entre población universitaria: representaciones sociales e implicaciones en temas ambientales en la Universidad de Antioquia. El Ágora USB, 14(1), 241-256. https:// doi.org/10.21500/16578031.128

Moscovici, S. (1979). El psicoanálisis su imagen y su público. Buenos Aires. Hemul. Ramírez, Y. (2014). Estudio comparativo de las representaciones sociales del cambio climático en estudiantes de licenciatura para la estructuración de pautas de 
comunicación educativa. Tesis para obtener el doctorado en Investigación Educativa. México: Universidad Veracruzana.

Rebich, S. y Gautier, C. (2005). Concept mapping to reveal prior knowledge and conceptual change in a mock summit course on global climate change. Journal of Geoscience Education, 53(4), 355-365. https://doi. org/10.5408/1089-9995-53.4.355

Sauvé, L. (1997). Pour une éducation liée à l'environnement. Montreal: Guérin.

Terrón E. y Bahena, D. (2015). Saberes del cambio climático de los universitarios y el camino pendiente de la educación ambiental. Trabajo presentado en el XIII Congreso Nacional de Investigación Educativa. Chihuahua, México.

Universidad Complutense de Madrid (2008). Actitudes, información e implicación de los jóvenes en el desarrollo sostenible de la ciudad de Madrid. Disponible en http://fongdcam.org/manuales/sostenibilidad/datos/ docs/1_ARTICULOS_Y_DOCUMENTOS_DE_REFERENCIA/M_ PARTICIPACION_CIUDADANA/juventud_y_medio_ambiente.pdf 